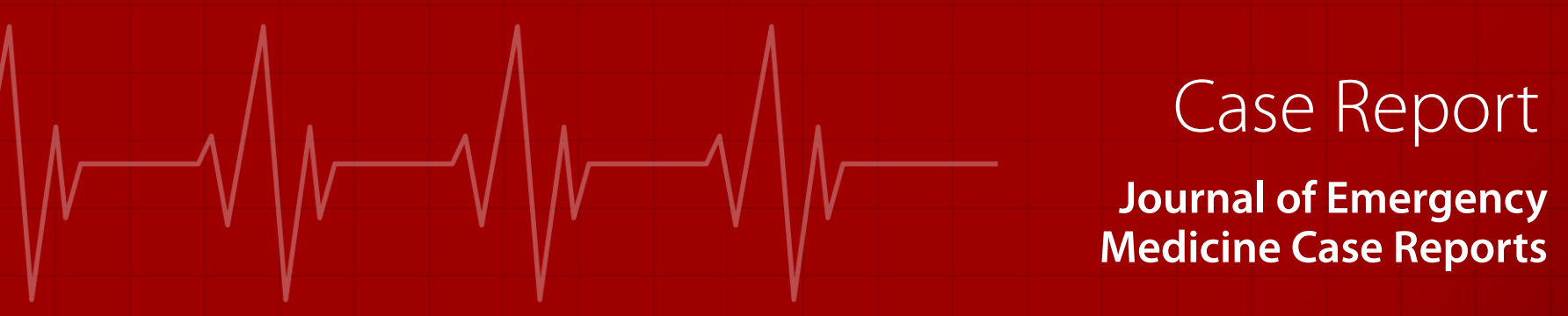

\title{
Chronic Licorice Consumption as a Rare Cause of Hypocalcemia
}

Eda Kan', Tuba Sarıaydın', Murat Dogan², Ali Kemal Erenler

${ }^{1}$ Department of Emergency Medicine, Hitit University Faculty of Medicine, Corum, Turkey

${ }^{2}$ Department of Internal Medicine, Hitit University Faculty of Medicine, Corum, Turkey

\begin{abstract}
Introduction: Licorice ingestion is well-described with its hypokalemic and hypertensive effects in the literature. To our knowledge, up to now, hypocalcemia due to licorice ingestion has not been described. Our aim is to create awareness for hypocalecemia concomittant with hypokalemia in patients with chronic licorice ingestion.

Case Report: A 51-year old male patient presented to our Emergency Department (ED) with complaint of numbness on his whole body. On anamnesis, it was determined that he has been consuming licorice to regulate his hypertension for 3 months. Laboratory analysis revealed hypokalemia and hypocalcemia. After replacement therapy, the patient was discharged from the hospital with full recovery.

Conclusion: Focusing on hypokalemia may lead to misdiagnosis of hypocalcemia in patients with chronic licorice ingestion. Physicians must be aware of other electrolyte disturbances not described in the literature yet.

Key Words: licorice ingestion, hypocalcemia, hypokalemia, emergency medicine
\end{abstract}

\section{Introduction}

Ingestion of licorice is a well-known reason for a syndrome mimicking mineralocorticoid excess, of which the pathophysiology is completely clarified. The characteristics of this syndrome are hypertension, hypokalaemia, alkalosis, low renin activity and hypoaldosteronism ${ }^{1}$. In this report, we present you a case with hypocalcemia accompanying hypokalemia in a patient with licorice consumption for hypertension regulation for 3 months. Our aim is to create awareness to hypocalcemia as a rare finding of chronic licorice consumption.

\section{Case Report}

A 51-year-old male patient was admitted to our Emergency Department (ED) due to generalized numbness. On admission he was hypertensive $(150 / 95 \mathrm{mmHg})$ with a heart rate of 84 beats/min, a saturation of $96 \%$ by probe and a temperature of $36,2^{\circ} \mathrm{C}$. On his medical history, hypertension for 10 years was determined. It was also determined that the patient has been consuming licorice for its antihypertensive properties for 3 months. Physical examination revealed apparent $\mathrm{Ch}-$ vostek's sign and Trousseau's sign. In laboratory analysis,
Calcium (Ca) was $6.7 \mathrm{mg} / \mathrm{dL}$ and Potassium $(\mathrm{K})$ was 3.5 $\mathrm{mmol} / \mathrm{L}$. A spot urine sample analysis showed urinary sodium of $122 \mathrm{mmol} / \mathrm{L}$ and potassium of $90 \mathrm{mmol} / \mathrm{L}$. Other parameters were normal. An ECG was obtained and it did not reveal any abnormalities. In order to exclude a intracranial pathology, a computerized tomography (CT) was obtained and it was also normal. Hypocalcemia and hypokalemia due to chronic licorice ingestion was considered as a predignosis and the patient was consulted with an internal medicine specialist and a nephrologist. According to their suggestions, $40 \mathrm{ml} \mathrm{K}$ in $500 \mathrm{cc}$ serum physiologique in 5 hours and 3 ampoules of $\% 10 \mathrm{Ca}$ in $5 \%$ dextrose in 2 hours were initiated. After 5 hours of ED observation, the patient was transferred to internal medicine ward. After 3-days follow-up with replacement therapy in the ward, symptoms of the patient and the laboratory values improved. The patient has been discharged with full recovery. The laboratory findings of the patient in the follow-up are summarized in Table 1.

\section{Discussion}

Licorice (Glycyrrhiza glabra L.) is one of the most widely used herb for ages. Licorice grows in the sub-tropical and warm temperate regions of the world, particularly in India and 
Table 1: Follow-up of $\mathrm{Ca}+$ and $\mathrm{K}+$ values.

\begin{tabular}{lcccc}
\hline & On admission & Day 1 & Day 2 & Day 3 \\
\hline $\mathrm{Ca}+(\mathrm{mg} / \mathrm{dL})$ & 6.7 & 6 & 7.4 & 7.8 \\
$\mathrm{~K}+(\mathrm{mmol} / \mathrm{L})$ & 3.5 & 2.9 & 3 & 3.6 \\
\hline
\end{tabular}

Mediterranean countries. Roots and stolon parts of licorice plants are used in mixed herbal preparations to promote digestion and vitality. Licorice root extract has been studied for its anti-cancer and anti-viral activities and healing of gastriculcers. Pharmacological investigations indicate that licorice root extracts have antioxidant, antibacterial and anti-inflammatory activities. The main ingredient of licorice roots glycyrrhizin or glycyrrhizic acid (GA), a triterpinoid saponin, is used for the control of cough, asthma, bronchitis, pepticulcer, arthritis and allergic reactions ${ }^{2}$. In our case, the patient declared that he used the herb to regulate hypertension.

Although hypokalemia is the most dangerous side effect of licorice consumption, the main adverse effect is hypertension. The USA Food and DrugAdministration (FDA) advises avoiding eating large amounts of black licorice at one time ${ }^{3}$. Our patient was also hypertensive on admission, however, we could not determine whether the hypertension was linked to licorice or the idiopatic hypertension in patient's medical history. It is an interesting example for misinformation since the patient uses a herb that causes hypertension to reduce hypertension. Unconscious consumptions of herbs may result in such life-threatening conditions.

Chronic licorice intoxication is a well-recognized cause of hypokalemia ${ }^{4}$. Additionally, it may cause hypokaliemic rhabdomyolysis, characterised by a sthenic deficit exclusively involving the distal muscles of the upper limbs and secondary to chronic glycyrrhizic acid intoxication, and by the absence of even ictal arterial hypertension ${ }^{5}$. Our patient complained of insensitiveness in whole body and laboratory findings revealed hypokalemia in concordance with the literature. However, in our case we also determined hypocalcemia which could not be explained with another pathology. To our knowledge, so far, hypocalcemic effect of licorice ingestion has not been identified in the literature.

Licorice withdrawal and potassium replacement are the mainstay of therapy, though caution is advised in the use of IV potassium and recommendations are made about the rate of infusion. The potassium-replacement therapy should consist of potassium administered in glucose free solutions, since glucose may cause a further decrease in potassium concentration and can reverse any beneficial effect of administered potassium, with the risk of precipitating arrhythmias, neuromuscular paralysis, and respiratory failure. The use of glucose-free solutions during IV administration of potassium should be regarded as an important therapeutic principle $^{4}$. In our case, in addition to $\mathrm{K}$ replacement, due to hypocalcemia, Ca replacement was required. A close follow-up and monitorization may be beneficial in such cases with multiple electrolyte abnormalities.

\section{Conclusion}

It is recommended for physicians working in the ED to be aware of the problems in electrolyte and blood pressure homeostasis that can occasionally occur with the excessive intake of licorice-containing products. In chronic licorice intake hypocalcemia accompanying hypokalemia must be kept in mind.

\section{References}

1. Janse $A$, van lersel $M$, Hoefnagels $W H$, Olde Rikker MG. The old lady who liked liquorice: hypertension due to chronic intoxication in a memory-impaired patient. Neth J Med. 2005; 63(4): 149-50.

2. Rohinishree YS, Negi PS. Effect of licorice extract on cell viability, biofilm formation and exotoxin production by Staphylococcus aureus. J Food Sci Technol. 2016; 53(2): 1092-100.

3. Caravaca-Fontan F, Martinez-Saez O, Delgado-Yague M, Yerovi E, Liano F. An Unexpected Cause of Severe Hypokalemia. Case Rep Nephrol. 2015; 2015: 957583.

4. Famularo G, Corsi FM, Giacanelli M. latrogenic worsening of hypokalemia and neuromuscular paralysis associated with the use of glucose solutions for potassium replacement in a young woman with licorice intoxication and furosemide abuse. Acad Emerg Med. 1999; 6(9): 960-4.

5. Barrella M, Lauria G, Quatrale R, Paolino E. Hypokaliemic rhabdomyolysis associated with liquoric eingestion: report of an atypical case. Ital J Neurol Sci. 1997; 18(4): 217-20. 